\title{
Cardiac Comorbidities in COPD Patients Explained Through HRV and Respiratory Indices
}

\author{
Daniel Romero ${ }^{1}$, Dolores Blanco-Almazan ${ }^{1,2,3}$ Willemijn Groenendaal ${ }^{4}$, Lien Lijnen ${ }^{5}$, \\ Christophe Smeets ${ }^{6}$, David Ruttens ${ }^{6}$, Francky Catthoor ${ }^{7,8}$, Raimon Jané ${ }^{1,2,3}$ \\ ${ }^{1}$ Institute for Bioengineering of Catalonia (IBEC-BIST), Barcelona, Spain \\ ${ }^{2}$ Biomedical Research Networking Center of Bioengineering, Biomaterials and Nanomedicine, \\ Barcelona, Spain \\ ${ }^{3}$ Universitat Politècnica de Catalunya $\cdot$ BarcelonaTech, Barcelona, Spain \\ 4 imec the Netherlands/Holst Centre, Eindhoven, the Netherlands \\ ${ }^{5}$ Hasselt University, Hasselt, Belgium ${ }^{6}$ Ziekenhuis Oost-Limburg, Genk, Belgium \\ ${ }^{7}$ imec, Heverlee, Belgium ${ }^{8}$ KU Leuven, Heverlee, Belgium
}

\begin{abstract}
Chronic obstructive pulmonary disease (COPD) patients exhibit depressed heart rate variability (HRV), while comorbidities may worsen the patients' prognosis. We investigated whether HRV analyis, clinical markers of disease severity and respiratory function, may explain the presence of cardiac-related comorbidities. Several HRV indices were evaluated in 46 COPD patients before a 6 minute walk test (6MWT). Maximum heart rate $\left(H R_{\max }\right)$ and walked distance (Dist) were measured during the test, while heart rate recovery (HRR) was estimated immediately afterwards. All these features and the patient characteristics were used to identify cardiac-related comorbidities (COPDco, $n=11)$. A logistic regression classifier with regularization was used for modeling and feature selection, while model assessment was performed by leave-oneout cross-validation. Only 4 features were needed to accurately identify comorbidities with overall performance metrics $A U C=84 \%$, sensitivity $=73 \%$ specificity $=83 \%$. The feature subset included the ratio given by the forced expiratory volume and the forced vital capacity ( $\left.F E V_{1} / F V C\right)$, the normalized HRR at minute 3, the Borg-scale of exertional dyspnea and the normalized LF power. These features could provide relevant information for early identification of cardiac comorbidities in COPD patients.
\end{abstract}

\section{Introduction}

Chronic obstructive pulmonary disease (COPD) is usually characterized by depressed heart rate variability (HRV), reflected through reduced vagal activity indices compared to healthy subjects [1]. If comorbidities are also present, they may accelerate the worsening of the patient diagnosis and prognosis, as occurs with cardiovascular diseases (CVD). This implies and increased risk of adverse cardio-respiratory events causing increased mortality and COPD-related hospitalisations [2], and thus a significant increase in public health cost.

The most common CVDs reported in patients with COPD are heart failure, peripheral vascular disease, arrhythmias and coronary artery disease. The standard diagnosis, delineated by the Global Initiative for Chronic Obstructive Lung Disease (GOLD), proposed a multidimensional classification score which is mainly focused on the condition of the lungs. That is, only pulmonary variables such as forced expiratory volume in one second $\left(\mathrm{FEV}_{1}\right)$ or arterial oxygen tension $\left(\mathrm{PaO}_{2}\right)$ are considered, and the treatment strategies usually only targets the lungs [3].

In order to improve the accuracy of the current approach, we decide to include other variables besides the pulmonary ones in COPD patients assessment. For this purpose, we investigated whether HRV markers, together with clinical markers of disease severity and respiratory function, may account for the presence of cardiac-related comorbidities in this particular population.

\section{Material and Methods}

\subsection{The six-minute walk test}

Fifty COPD patients were recruited at Ziekenhuis OostLimburg (Genk, Belgium) during their medical consultation or rehabilitation session. The patients were subjected to a $6 \mathrm{MWT}$ in which they walked along a $45-\mathrm{m}$ corridor for 6 minutes. The test allows to assess the functional exercise capacity in patients presenting with respiratory dis- 
eases [4]. The electrocardiogram (ECG) signal was continuously acquired 5 minutes before the test, during the entire walk, and 5 minutes during recovery. During the initial (resting) and final (recovery) phases, the patients were sitting in a wheel-chair.

Demographic and clinical data were collected at the hospital at the time of recruitment. Clinical data included the spirometry measures, in particular, the forced vital capacity (FVC) and the forced expiratory volume in one second $\left(\mathrm{FEV}_{1}\right)$. The study was approved by institutional medical ethics committee of the Ziekenhuis Oost-Limburg (reference: $18 / 0047 \mathrm{U})$. Patients provided written informed consent prior to study inclusion.

\subsection{ECG recording}

ECG recordings were acquired according to Lead II of Einthoven's triangle using a wearable research prototype device (imec the Netherlands, Eindhoven, the Netherlands) using standard $\mathrm{Ag} / \mathrm{AgCl}$ electrodes. The original sampling frequency of the ECG signals was $512 \mathrm{~Hz}$, but they resampled using spline interpolation to $1000 \mathrm{~Hz}$. The R-peaks were determined after QRS complexes detection using a wavelet-based method [5], followed by a visual inspection to reject ectopic and abnormal beats. Then, the temporal location of the R-peaks were used to generate the RR intervals time-series.

\subsection{Feature extraction}

\subsubsection{HRV analysis}

We evaluated conventional HRV indices including temporal and spectral markers from RR time-series corresponding to the initial 5-min resting segments. The temporal HRV indices included the standard deviation of normal RR intervals (SDNN), the percentage of successive $\mathrm{RR}$ intervals differing more than $50 \mathrm{~ms}$ (pNN50) and the root mean square of successive RR interval differences (RMSSD). In the case of the spectral indices, the RR timeseries were uniformly sampled via spline interpolation at $4 \mathrm{~Hz}$ before their calculation. When estimating the spectral density of the RR series, the Welch's periodogram was applied using a Hanning window. The calculated indices reflect the power of the components in the low-frequency (LF: 0.04-0.15 Hz) and high-frequency (HF: $0.15-0.40$ $\mathrm{Hz}$ ) bands of HRV analysis. These frequency bands are normally associated with the sympathetic and vagal activity modulated by the autonomic control. Both indices were also normalized by the total power, defined as the sum of the LF and HF power, thus obtaining the $\mathrm{LF}_{n o}$ and $\mathrm{HF}_{n o}$ markers. The ratio LF/HF completed the spectral analysis.

\subsubsection{HRR markers}

The autonomic control response during the post-walk recovery was assessed through the evaluation of the heart rate (HR) dynamic immediately after the 6MWT. Based on [6], we fitted on the HR time-series a biexponential equation and from the resulting signal, the heart rate recovery (HRR) marker were computed at specific time points. In particular, we computed the heart rate decay (i.e, the difference between the maximum and actual heart rate values) at the first, second and third minute of recovery, denoted here as $\mathrm{HRR}_{1}, \mathrm{HRR}_{2}$ and $\mathrm{HRR}_{3}$ respectively. Moreover, normalized HRR measures (in \%) were obtained, by dividing them with the $\mathrm{HR}$ achieved at the end of walk $\left(\mathrm{HR}_{\max }\right)$.

\subsection{Modeling}

Once all features were extracted from the different phases, the next step consisted of finding the best feature subset needed to identify comorbidities. To do this, we applied a selected prediction algorithm (logistic regression) with LASSO regularization [7]. This approach allows to penalize large model coefficients using the $\lambda$ hyperparameter, that controls the regularization strength. Besides, the value of $\lambda$ also serve to identify the most predictive features, and it can be selected based on prediction errors and $k$-fold cross-validation. This strategy has allowed to obtain sparse models while reducing overfitting.

The final feature subset selected by LASSO was entered in an ordinary logistic regression (OLS) model to obtain debiased LASSO coefficients. The resulting multivariate models were finally assessed by leave-one-out crossvalidation (LOOCV). Both sensitivity (Se) and specificity (Sp) metrics were reported together with the area under the curve (AUC) of the generated ROCs. Models with only respiratory function parameters and in combination with cardiac markers were tested for comparison purpose.

\subsection{Statistical analysis}

Results were expressed in mean \pm standard deviation (SD). Univariate statistical analysis was performed for individual parameters to find possible correlation with the presence of comorbidities. The Wilcoxon-Mann-Whitney test non-parametric test was applied for statistical comparison between patient groups. The level of significance was set to 0.05 for all the analysis. Data were processed and analyzed using custom MATLAB routines.

\section{Results}

\subsection{Study population}

Fifty COPD patients were recruited, 38 males and 12 females, and four patients were excluded due to the low 
signal-to-noise ratio of the ECG signals. The study population description is shown in Table 1.

Table 1. Demographic and anthropometric data for the study population.

\begin{tabular}{lc}
\hline \hline & $\mathrm{n}=46$ \\
\hline Male (Female) & $34(12)$ \\
Age $[\mathrm{yr}]$ & $65.00(60.00-69.00)$ \\
Height $[\mathrm{cm}]$ & $169.50(164.00-178.00)$ \\
BMI [kg/m $\left.{ }^{2}\right]$ & $24.85(22.27-29.04)$ \\
Smoker: Current $\quad 9(19.57 \%)$ \\
$\quad$ Former & $37(80.43 \%)$ \\
FVC \% pred & $86.45(72.20-110.90)$ \\
FEV ${ }_{1} \%$ pred & $52.15(42.80-68.60)$ \\
Cardiac comorbidity & $11(23.91 \%)$ \\
\hline \hline
\end{tabular}

Data are presented as median $\left(1^{\text {st }}-3^{r d}\right.$ quartile). BMI: body mass index; FVC: forced vital capacity; $\mathrm{FEV}_{1}$ : forced expiratory volume in one second.

\subsection{Univariate and multivariate analysis}

Univarite analysis: Patients with comorbidities were compared to those who did not present any cardiac disease at the moment of measurement. Figure 1 displays the box-plots of some features being significantly different among groups. Respiratory function parameters like the $\mathrm{FEV}_{1} / \mathrm{FVC}$ was significantly different $(61.2 \pm 11.6 \%)$ in the COPDco group compared to COPDnco patients $(45.2 \pm 12.3 \%), p=0.001$, while for $\mathrm{BS}_{\text {dispnea }}$, the values were $2.4 \pm 1.7$ and $4.3 \pm 2.2, p=0.007$, respectively. Regarding cardiac-related parameters, the normalized HRR evaluated at minute $3, \mathrm{HRR}_{3}$, was significantly faster in COPDco patients, with $23.7 \pm 5.9 \%$ vs $16.8 \pm 7.4 \%$, $p=0.005$. The remaining features did not reach significant differences between groups. However, all variables were included in the initial feature space used for identifying cardiac comorbitidies.

Multivariate analysis: Although a few variables reached significant differences among groups, all features were entered into the feature selection process performed by LASSO. The final selected $\lambda$ allowed to retain only 4 highly predictive features, including the $\mathrm{FEV}_{1} \mathrm{FVC}$, $\mathrm{HRR}_{n o}, \mathrm{BS}_{\text {dyspnea }}$ and $\mathrm{LF}_{n o}$, ordered by importance. This resulted in the best model with the highest classification performance while keeping model simplicity. Table 2 summarize the different model statistics.

From table 2, it can be observed that only two parameters $\left(\mathrm{FEV}_{1} / \mathrm{FVC}\right.$ and $\left.\mathrm{HRR}_{3}\right)$ were significant $(p<0.05)$ in the multivariate model. The positive sign of the coefficients and $\mathrm{ORs} \geq 1$ suggest these parameters as significant factors linked to cardiac comorbidities in this popula-

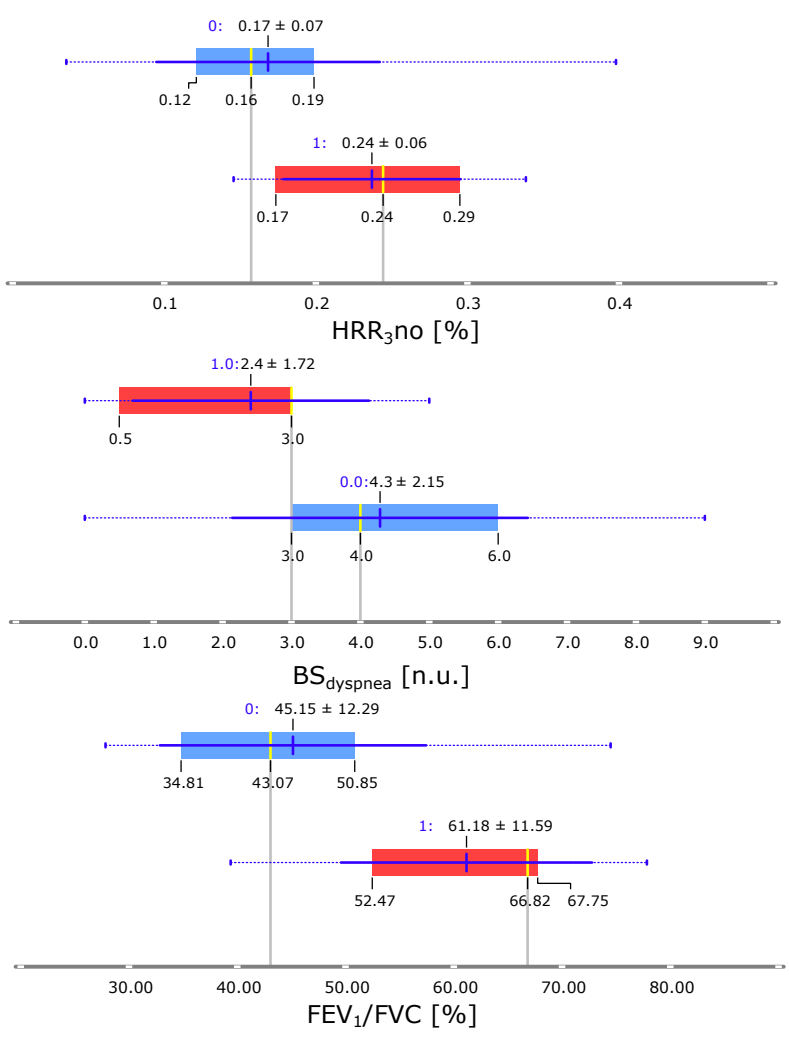

Figure 1. Boxplots of relevant parameters $\mathrm{HRR} 3_{n o}$, $\mathrm{BS}_{\text {dyspnea }}$ and $\mathrm{FEV}_{1} / \mathrm{FVC}$ comparing $\mathrm{COPD}_{c o}$ (red) and $\mathrm{COPD}_{n c o}$ (blue) patients. Numeric values represent mean $\pm \mathrm{SD}$, median (in yellow) and $1^{\text {st }}$ and $3^{\text {rd }}$ interquartile. Patients groups are represented by blue numbers.

Table 2. Model obtained for comorbidities detection.

\begin{tabular}{lcccc}
\hline \hline Feature & coeff & SE & OR $(95 \%$ CI $)$ & $p$ \\
\hline Intercept & -4.178 & 2.65 & $0.02(0.00-2.80)$ & 0.115 \\
$\mathrm{FEV}_{1} /$ FVC & 0.099 & 0.04 & $1.10(1.02-1.19)$ & $\mathbf{0 . 0 1 3}$ \\
$\mathrm{HRR}_{3}$ & 15.768 & 7.07 & $7 \mathrm{e} 6(6.70-7 \mathrm{e} 12)$ & $\mathbf{0 . 0 2 5}$ \\
$\mathrm{BS}_{\text {dyspnea }}$ & -0.776 & 0.41 & $0.46(0.21-1.03)$ & 0.057 \\
$\mathrm{LF}_{n o}$ & -0.056 & 0.03 & $0.95(0.89-1.00)$ & 0.056 \\
\hline
\end{tabular}

SE: standard error, OR: odd ratio, CI: confidence interval.

tion. Contrarily, although $\mathrm{BS}_{\text {dyspnea }}$ and $\mathrm{LF}_{n o}$ presented less significance coefficients, their changes would indicate a lower chance of having comorbidities.

Figure 2 (left column) shows the performance metrics obtained for the model in table 2, based on the receiver operating characteristic (ROC) curve. As expected, the AUC, Se and $\mathrm{Sp}$ values obtained for the training data is clearly superior to those obtained by LOOCV (right column), using the same feature set. Nevertheless, the latter provides a suitable model performance with respect to new observed data not seen during training. 

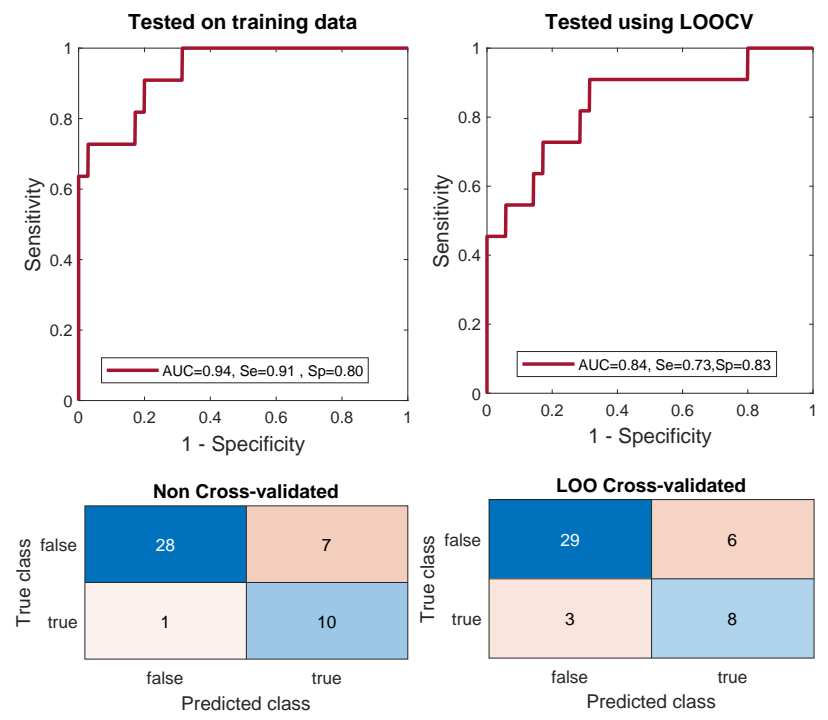

Figure 2. ROC curves obtained for the model tested on the training data (left) and by LOOCV (right). The corresponding confusion matrix are displayed at the bottom.

\section{Discussion and conclusions}

In this study we investigated a set of clinical parameters of respiratory functional capacity and cardiac-related markers evaluated in COPD patients. In particular, cardiac parameters, mostly related to HRV analysis, were included to better identify comorbidities in this population.

We first investigated the evaluated markers through univariate analysis to find those ones that better discriminate the presence of cardiac comorbidities. Two markers associated with lung functional capacity $\left(\mathrm{BS}_{\text {dyspnea }}\right.$ and $\mathrm{FEV}_{1} / \mathrm{FVC}$ ) and another with the heart rate dynamic during recovery $\left(\mathrm{HRR} 3_{n o}\right)$, were the most important to differentiate among the analyzed groups. From the multivariate analysis performed using the LASSO regularization, four predictive features including the three mentioned above, entered into the final selected model. In this case, the $\mathrm{HRR}_{n o}$ and $\mathrm{FEV}_{1} / \mathrm{FVC}$ remained most significant, and the model achieved an overall performance metrics of $\mathrm{AUC}=84 \%, \mathrm{Se}=73 \%, \mathrm{Sp}=83 \%$. These two markers were suggested to be the most relevant factors associated with comorbidities of cardiac origin in COPD patients.

Our results highlight the importance of considering different factors for a more complete assessment of COPD patients. Many of these patients may present comorbidities, and those of cardiac origin may worsen the patient prognosis and accelerate disease progression. Increased HR recovery after the 6MWT appears to be linked to cardiac comorbidities, although its presence has been associated with a better lung function and lower survival [8].

\section{Acknowledgments}

This project has received funding from the European Union's Horizon 2020 Research and Innovation Programme under the Marie Sklodowska-Curie grant agreement No. 846636. This work was also supported in part by the Universities and Research Secretariat from the Generalitat de Catalunya (GRC 2017 SGR 01770 and FIDGR), in part by the Agencia Estatal de Investigacion from the Spanish Ministry of Science, Innovation and Universities and the European Regional Development Fund (RTI2018 098472-B-I00), and in part by the CERCA Programme/Generalitat de Catalunya.

\section{References}

[1] Roque AL, Valenti VE, Massetti T, Da Silva TD, de Mello Monteiro CB, et al. Chronic obstructive pulmonary disease and heart rate variability: a literature update. International Archives of Medicine 2014;7(1):43.

[2] Mannino DM, Thorn D, Swensen A, Holguin F. Prevalence and outcomes of diabetes, hypertension and cardiovascular disease in copd. European Respiratory Journal 2008; 32(4):962-969.

[3] Agusti A, Noguera A, Sauleda J, Sala E, Pons J, Busquets X. Systemic effects of chronic obstructive pulmonary disease. European Respiratory Journal 2003;21(2):347-360.

[4] Holland AE, Spruit MA, Troosters T, Puhan MA, Pepin V, Saey D, et al. An official European Respiratory Society/American Thoracic Society technical standard: field walking tests in chronic respiratory disease. European Respiratory Journal dec 2014;44(6):1428 - 1446.

[5] Martinez JP, Almeida R, Olmos S, Rocha AP, Laguna P. A wavelet-based ecg delineator: evaluation on standard databases. IEEE Transactions on Biomedical Engineering April 2004;51(4):570-581. ISSN 1558-2531.

[6] Romero D, Béhar N, Mabo P, Hernández A. A biexponential approach for assessing parasympathetic reactivation after submaximal exercise. In 2017 Computing in Cardiology (CinC). ISSN 2325-887X, Sep. 2017; 1-4.

[7] Tibshirani R. Regression shrinkage and selection via the lasso. Journal of the Royal Statistical Society Series B Methodological 1996;58(1):267-288.

[8] Lange P, Marott JL, Vestbo J, Olsen KR, Ingebrigtsen TS, Dahl M, et al. Prediction of the clinical course of chronic obstructive pulmonary disease, using the new gold classification: a study of the general population. American Journal of Respiratory and Critical Care medicine 2012;186(10):975981.

Address for correspondence:

Daniel Romero

Ave. E. Maristany 16, 08019 Barcelona, Spain

dromero@ibecbarcelona.eu 